\title{
DISCRETE-TIME RISK-SENSITIVE FILTERS WITH NON-GAUSSIAN INITIAL CONDITIONS AND THEIR ERGODIC PROPERTIES
}

\author{
Subhrakanti Dey and Charalambos D. Charalambous
}

\begin{abstract}
In this paper, we study asymptotic stability properties of risk-sensitive filters with respect to their initial conditions. In particular, we consider a linear time-invariant systems with initial conditions that are not necessarily Gaussian. We show that in the case of Gaussian initial conditions, the optimal risksensitive filter asymptotically converges to a suboptimal filter initialized with an incorrect covariance matrix for the initial state vector in the mean square sense provided the incorrect initializing value for the covariance matrix results in a risk-sensitive filter that is asymptotically stable, that is, results in a solution for a Riccati equation that is asymptotically stabilizing. For non-Gaussian initial conditions, we derive the expression for the risk-sensitive filter in terms of a finite number of parameters. Under a boundedness assumption satisfied by the fourth order absolute moment of the initial state variable and a slow growth condition satisfied by a certain Radon-Nikodym derivative, we show that a suboptimal risk-sensitive filter initialized with Gaussian initial conditions asymptotically approaches the optimal risk-sensitive filter for nonGaussian initial conditions in the mean square sense. Some examples are also given to substantiate our claims.
\end{abstract}

KeyWords: Risk-sensitive estimantion, asymptotic stability, non-Gaussian, optimal filtering.

\section{INTRODUCTION}

Risk-sensitive filtering optimizes an exponential of quadratic (or more general convex) cost criterion. As opposed to $L_{2}$ filtering, risk-sensitive filtering penalizes the higher order moments of the estimation error energy, thus making the filters useful in uncertain plant and noise environments. It also allows a trade-off between optimal filtering for the nominal model case and the average noise situation, and robustness to worst case noise and model uncertainty by weighting the index of the exponential by a risk-sensitive parameter. For example, it has been shown in [13] that discrete-time risk-sensitive filters for hidden Markov models (HMM) with finite-discrete states perform better than standard HMM filters in situations involving uncertainties in the noise statistics. A more recent work Boel et al. [6] shows that such risk-sensitive filters

Manuscript received August 10, 2000; accepted November 30, 2000.

Subhrakanti Dey is with Department of Electrical and Electronic Engineering, University of Melbourne, Parkville, VIC 3052, Australia.

Charalambos D. Charalambous is with Department of Electrical Engineering, McGill University, Montreal, Canada H3A 2 A7. enjoy an error bound which is the sum of two terms, the first of which coincides with an upper bound on the error one would obtain if one knew exactly the underlying probability model, while the second term is a measure of the distance between the true and design probability models. Although risk-sensitive filtering was introduced for discrete-time linear systems in (Speyer et al. [23]), the term "risk-sensitive filtering" was introduced in (Dey and Moore [14]) and more general discrete-time nonlinear systems were treated, using similar techniques of (James et al. [17]) in the context of risk-sensitive control. Apart from the potential usefulness of risk-sensitive filters in uncertain environments, risk-sensitive problems, in the small noise-limit, have been shown to be closely related to estimation/control problems in a deterministic worst-case noise scenario given from a differential game $\left(H_{\infty}\right.$ estimation/control problems for linear systems) (Whittle [24,25], James et al. [17], Moore et al. [19] and Charalambous [8]).

It is well known that the mean of the conditional density of the state given the observations for a stochastic state space signal model achieves the minimum variance filter. For a linear Gaussian system with deterministic or Gaussian distributed initial conditions, the conditional density is Gaussian and given by its mean and covariance (which can be calculated off-line from a Riccati differen- 
tial or difference equation). This is also popularly known as a Kalman filter. On the other hand, the optimal estimation problem becomes an essentially nonlinear problem if the initial condition does not have a Gaussian distribution. However, for linear Gaussian systems, it has been shown (Makowski [18], Ocone and Pardoux [20]) that the optimal filter (or its density) can be given by a finite number of statistics, which constitute the optimal (in the minimum variance sense) filter for an augmented linear system. The initial condition is often not known and it is often unrealistic to assume that the initial condition has a Gaussian density. However, it has been shown in (Ocone and Pardoux [20]) (continuous-time), (Sowers and Makowski [22]) (discrete-time) that the conditional density filter forgets the initial condition asymptotically in an exponential rate. In other words, one can assume a Gaussian density for the initial condition and use a suboptimal Kalman filter which asymptotically becomes optimal, provided the actual density of the initial condition has finite first and second order moments. Exponential stability results for discrete-time filters have been shown in (Budhiraja and Ocone [7]) and for (Benes filters Benes [3]) in (Ocone [21]). Also, stability results for filters based on Lyapunov exponents have been explored in (Deylon and Zeitouni [15], Atar and Zeitouni [2] and Atar and Zeitouni [1]).

It is also well known that the optimal risk-sensitive filter for a discrete-time linear Gaussian system with a Gaussian initial condition is an $H_{\infty}$ filter (Speyer et al. [23], Dey and Moore [14]). Analogous results for continuoustime systems can be found in (Charalambous et al. [9], Dey et al. [12], Dey and Charalambous [11]). In the case of a non-Gaussian initial condition, the risk-sensitive estimation problem, as can be expected, becomes a nonlinear problem in general.

In this paper, we consider the problem of risksensitive estimation for discrete-time linear Gaussian timeinvariant systems with non-Gaussian initial conditions. Our objective is to study the effects of initial conditions on the risk-sensitive estimates and asymptotic stability or forgetting properties of such estimates with respect to initial conditions. We first consider the case of arbitrary Gaussian initial conditions, i.e., arbitrary initial covariance matrices (the mean of the Gaussian distribution is taken to be zero without loss of generality), a suboptimal risk-sensitive estimate (initialized with an incorrect covariance matrix) asymptotically approaches the optimal risksensitive estimate (initialized with the true covariance matrix) provided the incorrect initial covariance matrix results in a stabilizing solution of the $H_{\infty}$-like Riccati equation. The case with non-Gaussian initial conditions is slightly more complicated. We first derive an expression for the risk-sensitive estimate that is finite-dimensional, and a sum of two quantities, the first of which asymptotically approaches the risk-sensitive estimate for the Gaussian initial condition (with arbitrary but stabilizing initial cova- riance matrix) and the second term approaches zero asymptotically under a boundedness condition satisfied by the fourth order absolute moment of the initial state variable and a slow growth condition satisfied by a the fourth order moment of a certain Radon-Nikodym derivative. These convergence results are derived in the mean square sense.

In Section 2, we introduce the signal model, the risksensitive estimation problem and reformulate it under a new probability measure. In Section 3, we briefly present the optimal risk-sensitive filter for linear Gaussian systems with Gaussian initial conditions and show the asymptotic stability of these filters with respect to arbitrary Gaussian initial conditions in the mean square sense. Section 4 deals with non-Gaussian initial conditions where we first derive the optimal risk-sensitive filter using the information state approach and then we show the asymptotic mean square convergence properties of such filters with respect to their initial conditions. Section 5 presents some concluding remarks.

\section{SIGNAL MODEL}

Consider a probability space $(\Omega, \mathcal{F}, P)$ on which we define the following stochastic linear time-invariant discrete-time state space model:

$$
\begin{aligned}
& x_{k+1}=F x_{k}+G w_{k+1}, x_{0} \sim \Pi_{0}\left(x_{0}\right) \\
& y_{k}=H x_{k}+v_{k}
\end{aligned}
$$

Here $x_{k} \in \mathbb{R}^{n}, y_{k} \in \mathbb{R}^{p}, k \in \mathbb{N}$. The process noise $w_{k} \in \mathbb{R}^{n}$ and the measurement noise $v_{k} \in \mathbb{R}^{p}$ are i.i.d. Gaussian distributed with zero mean and covariance $I_{n}$ and $I_{p}$ respectively. Also, $G G^{*}=\Sigma_{w}>0 . \Pi_{0}$ is not necessarily Gaussian.

We assume that $x_{0}, w_{k}, v_{k}$ are mutually independent and that $(F, G)$ are stabilizable and $(F, H)$ are detectable.

Denote the filtration generated by the observation $\sigma$-algebra, namely, $\sigma\left\{y_{0}, y_{1}, \ldots, y_{k}\right\}$ as $\left\{y_{k}\right\}$, the filtration generated by $\sigma\left\{x_{0}\right\} \vee \sigma\left\{w_{1}, \ldots, w_{k-1}\right\}$ as $\left\{\mathcal{F}_{k}\right\}$ and the filtration generated by $\sigma\left\{y_{0}, \ldots, y_{k}\right\} \vee \sigma\left\{x_{0}\right\} \vee \sigma\left\{w_{0}, \ldots\right.$, $\left.w_{k-1}\right\}$ as $\left\{\mathcal{G}_{k}\right\}$.

\section{Risk-sensitive estimation}

We define the risk-sensitive estimation problem for the discrete-time system (1) as to obtain a $y_{k}$-measurable process $\hat{x}_{k} \in \mathbb{R}^{n}$ (assumed to be well-defined) such that

$$
\hat{x}_{k} \in \underset{\zeta}{\operatorname{argmin}} E\left[\exp \mu\left\{\sum_{m=0}^{k-1} l\left(x_{m}, \hat{x}_{m}\right)+l\left(x_{k}, \zeta\right)\right\} \mid y_{k}\right]
$$

Here, $E\left[\right.$.] denotes expectation under $P, \mu>0$ and $l: \mathbb{R}^{n} \times$ $\mathbb{R}^{n} \rightarrow \mathbb{R}$ is measurable in $(x, \hat{x})$ and continuous in $\hat{x}$ and is of the following form 


$$
l(x, \hat{x})=\frac{1}{2}(x-\hat{x})^{*}(x-\hat{x})
$$

Remark 2.1 Note above that while estimating $\hat{x}_{k}$, we do not obtain new estimates of $x_{m}, m<k$. Hence this is a strict filtering problem. However, we consider a sum of estimation error costs in the index of the exponential. Note that considering just the cost at one time point (instead of the sum) will give rise to a different optimization problem. In the linear Gaussian case, this results in a Kalman filter whereas a cost like (2) results in an $H_{\infty}$ filter. For more discussion on this, see (Moore et al. [19], Dey and Moore [13]).

Note also that to have a meaningful estimation problem as set out above, we need to make the following assumption:

Assumption 2.1 We assume that $\hat{x}_{k}$ exists for all $k \in \mathbb{N}$ and $\mu$ is small enough such that

$$
\begin{aligned}
& \lim _{T \rightarrow \infty} \frac{1}{T} \log E\left[\exp \mu\left\{\sum_{m=0}^{k-1} l\left(x_{m}, \hat{x}_{m}\right)\right\} \mid \vartheta_{T}\right] \leq c_{1}<\infty, \\
& \text { for some } c_{1}>0
\end{aligned}
$$

Remark 2.2 It is important to know when $c_{1}$ in the above assumption is finite, before evaluating $\hat{x}_{k}$ as defined by (2), (3). A useful guideline for this can be found in (Boel et al. [6]). The idea, in short, is to verify that for some small $\mu$, there exists a finite number $c_{1}^{s}$ such that $\log E\left[\exp \mu\left\{\sum_{m=0}^{T} l\left(x_{m}, \hat{x}_{m}^{s}\right)\right\} \mid \mathcal{Y}_{T}\right] \leq c_{1}^{s} T$ where $\hat{x}_{k}^{s}$ is some sub-optimal estimator obtained through some heuristics. For example, in the case where the signal model is linear Gaussian as described by (1) and the initial condition is Gaussian, one can use the Kalman filter (i.e., the conditional expectation filter) as the sub-optimal estimator $\hat{x}_{k}^{s}$ and show that such a finite $c_{1}^{s}$ exists. This will then automatically imply the existence of a finite $c_{1}$ as in Assumption 2.1

Next, we work under a probability measure $\bar{P}$ such that under $\bar{P},\left\{y_{k}\right\}$ is a sequence of i.i.d. Gaussian distributed random variables with zero mean and covariance $I_{p}$ and independent of $x_{k}$ (and hence $x_{0}$ ). Using a discretetime change of measure formula (note that the continuoustime counterpart is given by Girsanov's theorem), the risksensitive estimation can be re-formulated as

$$
\hat{x}_{k} \in \underset{\zeta}{\operatorname{argmin}} \bar{E}\left[\Lambda_{k} \exp \mu\left\{\sum_{m=0}^{k-1} l\left(x_{m}, \hat{x}_{m}\right)+l\left(x_{k}, \zeta\right)\right\} \mid \gamma_{k}\right]
$$

where $\Lambda_{k}=\Pi_{l=0}^{k} \exp \left(\left(H x_{k}\right)^{\prime} y_{k}-\frac{1}{2}\left(H x_{k}\right)^{\prime}\left(H X_{k}\right)\right)$. For details on this particular application of change of probability measure technique, see (Elliott et al. [16]) (discrete-time) and (Bensoussan [4], Dey et al. [12]) (continuous-time).

\section{DISCRETE-TIME RISK-SENSITIVE ESTIMATION WITH GAUSSIAN INITIAL CONDITION}

In this section, we present the risk-sensitive estimation results for discrete-time linear Gaussian systems with Gaussian initial conditions and study the asymptotic forgetting property of the estimates with respect to initial conditions. Without loss of generality (see Makowski [18]), we take the mean of the initial density to be zero. It is with respect to the covariance matrix of the initial state that we study the asymptotic convergence properties.

Consider the signal model (1). Also, suppose $x_{0} \sim N$ $(0, \Sigma)$. Suppose also that the following assumption is satisfied:

Assumption 3.1 There exists a bounded positive definite symmetric solution $\Sigma_{k}$ to the following Riccati difference equation:

$$
\begin{aligned}
& \Sigma_{k}^{-1}=H^{\prime} H+\left[\Sigma_{w}+F\left(\Sigma_{k-1}^{-1}-\mu I\right)^{-1} F^{\prime}\right]^{-1}, \\
& \Sigma_{0}=\left(H^{\prime} H+\Sigma^{-1}\right)^{-1}
\end{aligned}
$$

The following theorem summarizes the risk-sensitive estimation results for the linear Gaussian systems with Gaussian initial condition (for similar proofs, see (Dey and Moore [14])).

Theorem 3.1 Consider the signal model (1) and the risksensitive cost given by (2), (3). Suppose $x_{0} \sim N(0, \Sigma)$. Also suppose that Assumption 3.1 holds. The optimal risksensitive estimate $\hat{x}_{k}^{G}$ is then given by the following stochastic difference equation

$$
\begin{aligned}
& \hat{x}_{k}^{G}=F \hat{x}_{k-1}^{G}+\Sigma_{k} H^{\prime}\left(y_{k}-H F \hat{x}_{k-1}^{G}\right) \\
& \hat{x}_{0}^{G}=\left(H^{\prime} H+\Sigma^{-1}\right)^{-1} H^{\prime} y_{0}
\end{aligned}
$$

where $\Sigma_{k}$ satisfies (6).

Proof. A similar proof can be found in (Dey and Moore [14]) and is not repeated here.

Remark 3.1 Note that Assumption 3.1 is equivalent to the assumption that $\mu$ is small enough such that $\Sigma_{w}+F\left(\Sigma_{k-1}^{-1}-\right.$ $\mu I)^{-1} F^{\prime}>0, \forall_{k}>0$ (Speyer et al. [23]).

\section{Asymptotic optimality of discrete-time risk-sensi- tive filters with Gaussian initial conditions}

In this subsection, we present the results for the asymptotic optimality of the discrete-time risk-sensitive 
filters with respect to arbitrary Gaussian initial conditions. Without loss of generality (Makowski [18]), we take the mean of the Gaussian density to be 0 . It is well known (from $H_{\infty}$ filtering theory) that the solutions to the Riccati equation (6) are not necessarily stabilizing under the previous stabilizability and detectability assumptions (unlike Kalman filtering Riccati equations). If $\Sigma_{0}$ is chosen such that $\lim _{k \rightarrow \infty} \Sigma_{k}$ (assuming that the limit exists) is stabilizing, then we consider $\Sigma_{0}$ to be a candidate for an arbitrary initial condition for (6). We denote the set of such admissible initial choices for $\Sigma_{0}$ as $\mathcal{D}$.

The steady state solution $\Sigma_{\infty}$ is the solution to the following algebraic Riccati equation:

$$
\Sigma_{\infty}^{-1}=H^{\prime} H+\left[\Sigma_{w}+F\left(\Sigma_{\infty}{ }^{-1}-\mu I\right)^{-1} F^{\prime}\right]^{-1}
$$

In what follows, we will always consider initializing values for $\Sigma_{0}$ that result in a stabilizing solution $\Sigma_{\infty}$.

Consider the following suboptimal risk-sensitive estimate $\beta_{k}^{G}$ which satisfies the following stochastic difference equation:

$$
\begin{aligned}
& \beta_{k}^{G}=F \beta_{k-1}^{G}+Q_{k} H^{\prime}\left(y_{k}-H F \beta_{k-1}^{G}\right) \\
& \beta_{0}^{G}=Q_{0} H^{\prime} y_{0}
\end{aligned}
$$

where $Q_{k}$ satisfies the following Riccati difference equation:

$$
Q_{k}^{-1}=H^{\prime} H+\left[\Sigma_{w}+F\left(Q_{k-1}^{-1}-\mu I\right)^{-1} F^{\prime}\right]^{-1}, Q_{0} \in \mathcal{D}
$$

In other words, (9), (6) describe a suboptimal risksensitive estimate with an arbitrary initial covariance matrix $Q \in \mathcal{D}$. We will show that $\beta_{k}^{G}$ converges to $\hat{x}_{k}^{G}$ in the mean square sense, that is as $k \rightarrow \infty, E\left|\hat{x}_{k}^{G}-\beta_{k}^{G}\right|^{2} \rightarrow 0$.

In order to be able to derive the intended asymptotic stability results, we modify Assumption 3.1 and assume the following is true:

Assumption 3.2 There exists a bounded symmetric positive definite solution $\Sigma_{k}$ to (6) for all $k>0$, where $\Sigma_{0} \in \mathcal{D}$ such that $\lim _{k \rightarrow \infty} \Sigma_{k}=\Sigma_{\infty}$ exists and $\rho\left(F-\Sigma_{\infty} H^{\prime} H F\right)<1$ where $\rho(A)$ is the spectral radius of the matrix $A$.

At this stage it is important to note that (except for the scalar case) obtaining a necessary and sufficient condition bounding $\mu$ such that Assumption 3.2 holds is hard. However, it is likely to hold for a small enough $\mu$, in analogy to results for $H_{\infty}$-like Riccati equations. Instead, we give an example of a system for which Assumption 3.2 is satisfied.

Example. Consider (1). Choose $F=\left[\begin{array}{ll}-0.8 & 0.9 \\ -0.2 & 0.7\end{array}\right]$ and $H=$ [0.8 0.1], $G=I_{2}$. Clearly, $(F, G)$ is completely stabilizable and $(F, H)$ is completely detectable. For $\mu=0.2$, one can work out that in this case $\mathcal{D}$ is nonempty that is for many choices of $\Sigma_{0}, \Sigma_{\infty}$ exists and is a positive definite symmetric matrix with $\rho\left(F-\Sigma_{\infty} H^{\prime} H F\right)<1$. In particular, $\Sigma_{\infty}=$ $\left[\begin{array}{ll}0.9531 & 0.2968 \\ 0.2968 & 1.5546\end{array}\right]$ and $\rho\left(F-\Sigma_{\infty} H^{\prime} H F\right)=0.4132$. There are also other choices of $\mu$ for which Assumption 3.2 holds true for this example

Remark 3.2 Assumption 3.2 implies that the following discrete-time unforced time-varying linear system

$$
\Psi_{k+1}=\left(F-\Sigma_{k} H^{\prime} H F\right) \Psi_{k}, \Psi_{0}=I
$$

is exponentially stable. This follows from the fact that (see Sowers and Makowski [22]) $\lim _{k \rightarrow \infty} \frac{1}{k} \ln \lambda_{\max }\left(\Psi_{k}^{\prime} \Psi_{k}\right) \leq 2 \ln$ $\rho\left(F-\Sigma_{\infty} H^{\prime} H F\right)$.

Based on the above discussions, we have the following two propositions which we state without proof:

Proposition 3.1 There exist $\left|\ln \rho\left(F-\Sigma_{\infty} H^{\prime} H F\right)\right|>\rho>0$, $M_{\sigma}^{1}>0$ such that

$$
\left\|\Psi_{k} \Psi_{j}^{-1}\right\| \leq M_{\sigma}^{1} \exp (-\sigma(k-j)), \forall k>j \geq 0
$$

Proposition 3.2 There exists a $\sigma>0, M_{\sigma}^{2}>0, k_{0} \geq 0$ such that

$$
\left\|\Sigma_{k}-Q_{k}\right\| \leq M_{\sigma}^{2} \exp (-\sigma k), \forall k \geq k_{0}
$$

For similar proofs in continuous-time literature, see (Ocone and Pardoux [20]) and the references therein. Related results in monotonicity and stability properties of discrete-time Riccati equations can be found in Bitmead et al. [5].

In other words, it follows that both $\lim _{k \rightarrow \infty} Q_{k} \rightarrow \Sigma_{\infty}$, $\lim _{k \rightarrow \infty} \Sigma_{k} \rightarrow \Sigma_{\infty}$ exponentially fast and also that the unforced linear system

$$
\widetilde{\Psi}_{k+1}=\left(F-Q_{k} H^{\prime} H F\right) \widetilde{\Psi}_{k}
$$

is exponentially stable.

We now present the main results of this section in the following theorem and the subsequent corollary. We do not include the proofs since they are very similar to the proofs for asymptotic stability for conditional density filters with arbitrary Gaussian initial conditions (Sowers and Makowski [22], Ocone and Pardoux [20]). These results essentially follow from Propositions 3.1, 3.2 and the exponential stability of (14). 
Theorem 3.2 Consider the risk-sensitive optimization problem given by (2), (3) with $x_{0} \sim N(0, \Sigma)$. Consider also the evolution equations for the optimal and suboptimal estimates given by (7), (9) and the associated Riccati difference equations (6), (10). Suppose Assumptions 2.1, 3.2 holds. Then,

$$
\lim _{k \rightarrow \infty} E\left|\hat{x}_{k}^{G}-\beta_{k}^{G}\right|^{2} \rightarrow 0
$$

One can also obtain the following corollary which specializes the previous theorem to the case of zero initial conditions for the risk-sensitive estimate and the Riccati equation (6):

Corollary 3.1 Consider the risk-sensitive optimization problem given by (2), (3) with $x_{0} \sim N(0, \Sigma)$. Consider also the evolution equations for the optimal risk-sensitive estimate given by (7), (6). Consider the following suboptimal risk-sensitive estimate given by

$$
\begin{aligned}
& \tilde{\beta}_{k}^{G}=F \tilde{\beta}_{k-1}^{G}+\tilde{\Sigma}_{k} H^{\prime}\left(y_{k}-H F \tilde{\beta}_{k-1}^{G}\right) \\
& \tilde{\beta}_{0}^{G}=0
\end{aligned}
$$

where $\widetilde{\Sigma}_{k}$ satisfies the following Riccati difference equation:

$$
\widetilde{\Sigma}_{k}^{-1}=H^{\prime} H+\left[\Sigma_{w}+F\left(\widetilde{\Sigma}_{k-1}^{-1}-\mu I\right)^{-1} F^{\prime}\right]^{-1}, \widetilde{\Sigma}_{0}=0
$$

Suppose Assumptions 3.2, 2.1 hold. Then,

$$
\lim _{k \rightarrow \infty} E\left|\hat{x}_{k}^{G}-\tilde{\beta}_{k}^{G}\right|^{2} \rightarrow 0
$$

Similarly,

$$
\lim _{k \rightarrow \infty} E\left|\beta_{k}^{G}-\tilde{\beta}_{k}^{G}\right|^{2} \rightarrow 0
$$

Remark 3.3 Note that $\tilde{\Sigma}_{0}=0$ implies $\tilde{\Sigma}_{1}=\left[H^{\prime} H+\Sigma_{w}^{-1}\right]^{-1}$ and we assume $\tilde{\Sigma}_{k}>0, \forall k>0$.

\section{DISCRETE-TIME RISK-SENSITIVE ESTIMATION WITH NON-GAUSSIAN INITIAL CONDITIONS}

In this section, we first derive the optimal risksensitive estimate for discrete-time linear time-invariant systems with non-Gaussian initial conditions. We derive a recursive update formula for a modified information state and express the optimal risk-sensitive estimate as a function of the parameters of the information state and the non-Gaussian distribution of the initial condition. Throughout this section, we assume that $x_{0} \sim \Pi_{0}\left(x_{0}\right)$, where $\Pi_{0}\left(x_{0}\right)$ is not Gaussian but has zero mean and satisfies certain properties. We will make the formal assumptions later on.
Also, the superscript $N G$ will stand for estimates with nonGaussian initial condition.

Now, we define the risk-sensitive information state conditioned on the initial state. Note that this is a slightly modified definition than the one used in (Dey and Moore [14]).

Definition 4.1 Define the unnormalized conditional measure $q_{k}(x, \xi)$ where

$q_{k}(x, \xi) d x=\bar{E}\left[\Lambda_{k} \exp \left(\mu \sum_{l=0}^{k-1} l\left(x_{l}, \hat{x}_{l}\right)\right) I\left(x_{k} \in d x\right) \mid \vartheta_{k}, x_{0}=\xi\right]$

$q_{0}(x, \xi)=\exp \left[-\frac{1}{2} \xi^{\prime} H^{\prime} H \xi+\xi^{\prime} H^{\prime} y_{0}\right] \delta(x-\xi)$

Remark 4.1 Note that the risk-sensitive information state defined in (Dey and Morre [14]) can be written as $q_{k}(x)$ (which is only conditioned on $y_{k}$ ) where

$$
q_{k}(x)=\int_{\mathbb{R}^{n}} q_{k}(x, \xi) \Pi_{0}(\xi) d \xi
$$

Using the Definition 4.1, one can easily prove the following Lemma:

Lemma 4.1 The information state $q_{k}(x, \xi)$ obeys the following recursive equation:

$$
\begin{aligned}
q_{k}(x, \xi)= & Z_{w} \exp \left[(H x)^{\prime} y_{k}\right. \\
& \left.-\frac{1}{2}(H x)^{\prime}(H x)\right] \int_{\mathbb{R}^{n}} \exp \left[-\frac{1}{2}(x-F z)^{\prime} \Sigma_{w}^{-1}(x-F z)\right] \\
& \exp \left[\frac{\mu}{2}\left(z-\hat{x}_{k-1}\right)^{\prime}\left(z-\hat{x}_{k-1}\right)\right] q_{k-1}(z, \xi) d z
\end{aligned}
$$

where $Z_{w}=\frac{1}{\left(2 \pi\left|\Sigma_{w}\right|\right)^{\frac{n}{2}}}$.

Proof. The proof simply follows from Definition 4.1. A similar proof can be found in (Dey and Moore [14]) and is not repeated here.

It also follows from the Definition 4.1 that the optimal risk-sensitive estimate is given by

$$
\hat{x}_{k} \in \underset{\zeta}{\operatorname{argmin}} \int_{I^{n}} \int_{I^{n}} q_{k}(x, \xi) \exp (\mu l(x, \zeta)) \Pi_{0}(\xi) d x d \xi
$$

It is obvious from the above Definition 4.1 that the information state achieves an expression similar to that for the information state with a known initial state vector. It is also well known that for known initial state vectors, the information state achieves an unnormalized Gaussian ex- 
pression for linear Gaussian systems. The proof follows by induction. We can use similar proof techniques to prove the following Theorem:

Theorem 4.1 The risk-sensitive information state defined as the unnormalized conditional measure in Definition 4.1 for the linear time invariant system (1) is given by

$$
q_{k}(x, \xi)=s_{k}(\xi) \exp \left(-\frac{1}{2}\left(x-m_{k}(\xi)\right)^{\prime} \widetilde{\Sigma}_{k}^{-1}\left(x-m_{k}(\xi)\right)\right)
$$

where $\widetilde{\Sigma}_{k}$ satisfies the Riccati difference equation (17) such that $\widetilde{\Sigma}_{k}>0, \forall_{k}>0, V_{k-1}^{-1}=F^{\prime} \Sigma_{w}^{-1} F+\widetilde{\Sigma}_{k-1}^{-1}-\mu I>0, \forall k$ $>1$ and $m_{k}(\xi)$ is given by the following equations:

$$
\begin{aligned}
& m_{k}(\xi)=\beta_{k}^{N G}+\Phi_{k} \xi, m_{0}(\xi)=\xi \\
& \widetilde{\Sigma}_{k}^{-1} \beta_{k}^{N G}=\Sigma_{w}^{-1} F V_{k-1}\left(\widetilde{\Sigma}_{k-1}^{-1} \beta_{k-1}^{N G}-\mu \hat{x}_{k-1}^{N G}\right)+H^{\prime} y_{k}, \beta_{0}^{N G}=0
\end{aligned}
$$

$\Phi_{k}$ is given by

$$
\Phi_{k}=\left[F-\widetilde{\Sigma}_{k} H^{\prime} H F+\mu \widetilde{\Sigma}_{k} \Sigma_{w}^{-1} F V_{k-1}\right] \Phi_{k-1}, \Phi_{0}=I
$$

Also $s_{k}(\xi)$ is given by

$$
\begin{aligned}
s_{k}(\xi)= & \gamma_{k} \exp \left[-\frac{1}{2} \xi^{\prime} L_{k} \xi+\xi^{\prime} \rho_{k}^{N G}\right] \\
\gamma_{k}= & \gamma_{k-1} Z_{k} S_{k}\left(\hat{x}_{k-1}, \beta_{k-1}, y_{k}\right), \gamma_{0}=1 \\
L_{k}= & L_{k-1}+\Phi_{k-1}^{\prime}\left(\widetilde{\Sigma}_{k-1}^{-1}-\widetilde{\Sigma}_{k-1}^{-1} V_{k-1} \widetilde{\Sigma}_{k-1}^{-1}\right) \Phi_{k-1} \\
& -\Phi_{k}^{\prime} \widetilde{\Sigma}_{k}^{-1} \Phi_{k}, L_{0}=H^{\prime} H \\
\rho_{k}^{N G}= & \rho_{k-1}^{N G}+\Phi_{k}^{\prime} \tilde{\Sigma}_{k}^{-1} \beta_{k}^{N G}-\Phi_{k-1}^{\prime}\left(\widetilde{\Sigma}_{k-1}^{-1}-\widetilde{\Sigma}_{k-1}^{-1} V_{k-1} \tilde{\Sigma}_{k-1}^{-1}\right) \beta_{k-1}^{N G} \\
& -\mu \Phi_{k-1}^{\prime} \widetilde{\Sigma}_{k-1}^{-1} V_{k-1} \hat{x}_{k-1}^{N G} \\
\rho_{0}^{N G}= & H^{\prime} y_{0}
\end{aligned}
$$

where $Z_{k}$ is a deterministic constant and $S_{k}\left(\hat{x}_{k-1}, \beta_{k-1}, y_{k}\right)$ is a function involving exponential of quadratic expressions of $\hat{x}_{k-1}, \beta_{k-1}, y_{k}$.

Proof. First of all, one can obtain an unnormalized Gaussian expression like (24) for $q_{1}(x, \xi)$ using the expression for $q_{0}$ $(x, \xi)$ given in (20). $q_{0}(x, \xi)$ also gives us the expressions for $\widetilde{\Sigma}_{0}, \beta_{0}^{N G}, \Phi_{0}, \gamma_{0}, L_{0}, \rho_{0}^{N G}$. One can then apply the method of induction to obtain the expression for $q_{k}(x, \xi)$ for any $k$ using Lemma 4.1. In view of the fact we are considering a linear Gaussian system with exponential of quadratic cost, the mean of the information state (as a function of $\xi$ ) naturally assumes an affine structure like that given in (25) (this approach is similar to that in Bensoussan [4]). $\beta_{k}^{N G}$, $\rho_{k}^{N G}, \hat{x}_{k}^{N G}$ bear the superscript $N G$ to denote that we are dealing with non-Gaussian initial conditions.

The recursive expressions for $\beta_{k}^{N G}, \widetilde{\Sigma}_{k}, \gamma_{k}, L_{k}$ and $\rho_{k}^{N G}$ are obtained equating two sides of (22) and expressing the right hand side of (22) in the form of the left hand side.

Remark 4.2 Note that the above theorem expresses the information state in terms of finite number of parameters $\beta_{k}^{N G}, \widetilde{\Sigma}_{k}, \gamma_{k}, L_{k}$ and $\rho_{k}^{N G}$. Also, $\Phi_{k}, \widetilde{\Sigma}_{k}$ and $L_{k}$ can be calculated off-line.

Also note that $\widetilde{\Sigma}_{0}=0$ merely implies that the initial condition is known.

One can now apply the above theorem to obtain the expression for the optimal risk-sensitive estimate using (23), which we state in the following theorem.

Theorem 4.2 Consider the linear time-invariant system given by (1). Consider also the cost objective given by (2) , (3). Suppose $x_{0} \sim \Pi_{0}\left(x_{0}\right)$. Then the optimal risk-sensitive estimate denoted by $\hat{x}_{k}^{N G}$ is given by

$$
\hat{x}_{k}^{N G}=\beta_{k}^{N G}+\Phi_{k} D_{k}\left(N_{k}^{N G}\right)
$$

where $D_{k}\left(N_{k}^{N G}\right)$ is given by

$$
D_{k}\left(N_{k}^{N G}\right)=\frac{\int_{\mathbb{R}^{n}} \xi \exp \left[-\frac{1}{2} \xi^{\prime} M_{k} \xi+\xi^{\prime} N_{k}^{N G}\right] \Pi_{0}(\xi) d \xi}{\int_{\mathbb{R}^{n}} \exp \left[-\frac{1}{2} \xi^{\prime} M_{k} \xi+\xi^{\prime} N_{k}^{N G}\right] \Pi_{0}(\xi) d \xi}
$$

and $M_{k}, N_{k}^{N G}$ are given by

$$
\begin{aligned}
M_{k}= & L_{k}+\Phi_{k}^{\prime} \widetilde{\Sigma}_{k}^{-1} \Phi_{k}-\Phi_{k}^{\prime} \widetilde{\Sigma}_{k}^{-1}\left(\widetilde{\Sigma}_{k}^{-1}-\mu I\right)^{-1} \widetilde{\Sigma}_{k}^{-1} \Phi_{k}, M_{0}=L_{0}-\mu I \\
N_{k}^{N G}= & \rho_{k}^{N G}-\Phi_{k}^{\prime} \widetilde{\Sigma}_{k}^{-1} \beta_{k}^{N G}+\Phi_{k}^{\prime} \tilde{\Sigma}_{k}^{-1}\left(\widetilde{\Sigma}_{k}^{-1}-\mu I\right)^{-1} \widetilde{\Sigma}_{k}^{-1} \beta_{k}^{N G} \\
& -\mu \Phi_{k}^{\prime} \widetilde{\Sigma}_{k}^{-1}\left(\widetilde{\Sigma}_{k}^{-1}-\mu I\right)^{-1} \hat{x}_{k}^{N G} \\
N_{0}^{N G}= & \rho_{0}^{N G}-\mu \hat{x}_{0}^{N G}
\end{aligned}
$$

Proof. The proof follows easily by using (23) along with the expression for $q_{k}(x, \xi)$ given by (24), (26). Differentiating with respect to $\hat{x}_{k}^{N G}$ and equating the derivative equal to zero, some algebraic manipulations result in (29), (30). The fact that the cost function is convex and approaches $\infty$ as $\left|\hat{x}_{k}^{N G}\right| \rightarrow \infty$, implies that the solution is a minimum and the desired solution. It also guarantees the existence of $N_{k}^{N G}, \forall k$ from above.

Remark 4.3 Note that the difficulty in obtaining a closed form expression for $\hat{x}_{k}^{N G}$ is that it is given by an implicit 
equation. This makes the analysis for asymptotic optimality of such estimates difficult and to simplify the analysis, we make certain assumptions in the next subsection. Although these assumptions are sufficient to guarantee the asymptotic optimality of risk-sensitive filters with respect to non-Gaussian initial conditions, it is essentially hard to verify some of the assumptions in practice. However, for $\mu=0$, one can solve the implicit equations explicitly to obtain solutions for risk-neutral estimation and similar results as in (Makowski [18]) can be obtained.

Note above that one can express $D_{k}\left(N_{k}^{N G}\right)$ as the conditional mean of $x_{0}$ under a different probability measure $\hat{P}$ such that $\frac{d \hat{P}}{d P}=\bar{\Lambda}_{k}$ where $\bar{\Lambda}_{k}$ is a $\left\{\mathscr{Y}_{k} \vee \sigma\left\{x_{0}\right\}\right\}$ adapted process given by

$$
\bar{\Lambda}_{k}=\frac{\exp \left[-\frac{1}{2} x_{0}^{\prime} M_{k} x_{0}+x_{0}^{\prime} N_{k}^{N G}\right]}{\int_{\mathbb{R}^{n}} \exp \left[-\frac{1}{2} \xi^{\prime} M_{k} \xi+\xi^{\prime} N_{k}^{N G}\right] \Pi_{0}(\xi) d \xi}
$$

Defining $E_{x_{0}}$ to be the expectation operator under measure $P$ with respect to the initial condition $x_{0}, D_{k}\left(N_{k}^{N G}\right)$ $=E_{x_{0}}\left[x_{0} \bar{\Lambda}_{k} \mid \mathcal{Y}_{k}\right]$ and also, $E_{x_{0}}\left[\bar{\Lambda}_{k}\right]=1$.

\section{Asymptotic optimality of risk-sensitive filters for non-Gaussian initial conditions}

In this section, we present the results on the mean square asymptotic convergence of the optimal risk-sensitive estimate to a suboptimal risk-sensitive estimate with a Gaussian initial condition with zero mean and arbitrary covariance matrix $Q \in \mathcal{D}$ (defined as $\beta_{k}^{G}$ in the previous Section).

Before presenting the main theorem on the convergence result, we make the following assumptions:

Assumption 4.1 $\mu$ is chosen small enough and $\Pi_{0}($.$) has$ such regularity properties that $\int_{\mathbb{R}^{n}} \exp \left[-\frac{1}{2} \xi^{\prime} M_{k} \xi+\right.$ $\left.\xi^{\prime} N_{k}^{N G}\right] \Pi_{0}(\xi) d \xi$ is well-defined for all $k$.

Example. For the example discussed in Section 3.1 with $\mu=0.2$, it can be worked out that $\lim _{k \rightarrow \infty} M_{k}=M_{\infty}$ exists and is positive definite symmetric. In fact, as evaluated from (30), $M_{k}$ is positive definite symmetric for all $k$ for $\mu$ $=0.2$. We can certainly find examples of non-Gaussian densities $\Pi_{0}($.) for which Assumption 4.1 holds in this case. One such example is

$$
\Pi_{0}(\xi)=\frac{1}{4(\Gamma(1 / 4))^{2}} \exp \left(-\xi_{1}^{4}-\xi_{2}^{4}\right)
$$

where $\Gamma(x)=\int_{0}^{\infty} t^{x-1} \exp (-t) d t$ and $\xi=\left(\xi_{1} \xi_{2}\right)^{\prime}$. Note however that the requirement of $M_{k}$ being a positive definite symmetric matrix for all $k$ is certainly not necessary but only a sufficient condition for Assumption 4.1 to hold for the above example.

Denote $F-\widetilde{\Sigma}_{k} H^{\prime} H F+\mu \widetilde{\Sigma}_{k} \Sigma_{w}^{-1} F V_{k-1} \triangleq A_{k}$. Then the existence of $\lim _{k \rightarrow \infty} A_{k}=A_{\infty}$ follows from the fact that $\lim _{k \rightarrow \infty} \tilde{\Sigma}_{k}=\tilde{\Sigma}_{\infty}$ exists.

\section{Assumption $4.2 \rho\left(A_{\infty}\right)<1$.}

Example. Again, for the same example as in Section 3.1, one can work out that for $\mu=0.2, \rho\left(A_{\infty}\right)=0.6211<1$.

Remark 4.4 Obviously, Assumption 4.2 guarantees that the following linear time-varying unforced linear system (see (26)):

$$
\Phi_{k}=\left[F-\widetilde{\Sigma}_{k} H^{\prime} H F+\mu \widetilde{\Sigma}_{k} \Sigma_{w}^{-1} F V_{k-1}\right] \Phi_{k-1}, \Phi_{0}=I
$$

is exponentially stable, i.e., there exist $\left|\ln \rho\left(A_{\infty}\right)\right|>\sigma_{1}>0$, $M_{\sigma_{1}}>0$ such that

$$
\left\|\Phi_{k}\right\| \leq M_{\sigma_{1}} \exp \left(-\sigma_{1} k\right)
$$

Assumption 4.3 There exists a $0<M_{x}<\infty$ such that $E$ $\left[\left|x_{0}\right|^{4}\right]<M_{x}$.

Remark 4.5 Note that this assumption is satisfied for the example given by (32).

Assumption 4.5 There exist $M_{d}>0$ and $0<\sigma_{d}<\sigma_{1}$ for some $0<\sigma_{1}<\left|\ln \rho\left(A_{\infty}\right)\right|$ such that $\Lambda_{k}^{-}$is a $\left\{y_{k} \vee \sigma\left\{x_{0}\right\}\right\}$ adapted process where $\sup _{k} E\left[\Lambda_{k}^{-4}\right] \leq M_{d} \exp \left(4 \sigma_{d} t\right)$.

Remark 4.6 Note that Assumption 4.3 and Assumption 4 . 4 together imply that $E\left|D_{k}\left(N_{k}^{N G}\right)\right|^{2} \leq M_{z} \exp \left(2 \sigma_{d} t\right), \forall k \in \mathbb{N}$ where $M_{z}>0$ is a constant. To see this, note that $\left|D_{k}\left(N_{k}^{N G}\right)\right|^{2}$ $\leq E_{x_{0}}\left[\left|x_{0}\right|^{2} \bar{\Lambda}_{k}^{2} \mid \mathcal{Y}_{k}\right]$ from Jensen's inequality. Hence $E\left|D_{k}\left(N_{k}^{N G}\right)\right|^{2} \leq E\left[\left|x_{0}\right|^{2} \bar{\Lambda}_{k}^{2}\right] \sqrt{E\left[\left|x_{0}\right|^{4}\right]} \sqrt{E\left[\Lambda_{k}^{4}\right]}$ where the last step follows from Schwartz's inequality. Now, using Assumption 4.3 and Assumption 4.4, it follows that $E\left|D_{k}\left(N_{k}^{N G}\right)\right|^{2} \leq M_{z} \exp \left(2 \sigma_{d} t\right), \forall k \in \mathbb{N}$. One can possibly look for a sufficient condition by imposing regularity properties on $\Pi_{0}($.$) and boundedness properties on the$ process $N_{k}^{N G}$ such that Assumption 4.4 is satisfied. But due to the complicated nature of the process $N_{k}^{N G}$ we postpone such investigation for the time being. However, it is clearly seen that Assumption 4.4 is not that restrictive since it allows an exponential growth (slow enough). 
With the above assumptions and Assumption 3.2 holding, one can summarize the main result of this section in the following theorem:

Theorem 4.3 Consider the signal model (1) where $x_{0} \sim \Pi_{0}$ $\left(x_{0}\right), \Pi_{0}$ being non-Gaussian. Consider also the risksensitive estimation problem given by (2),(3). Suppose Assumptions 3.2, 4.1, 4.2, 4.3 and 4.4 hold. Then the optimal risk-sensitive estimate given by (28), (29), (30) asymptotically approaches the suboptimal risk-sensitive estimate given by (16), (17) in the mean square sense, i.e.,

$$
\lim _{k \rightarrow \infty} E\left|\hat{x}_{k}^{N G}-\tilde{\beta}_{k}^{G}\right|^{2} \rightarrow 0
$$

Proof. Note that one can write

$$
\hat{x}_{k}^{N G}-\tilde{\beta}_{k}^{G}=\left(\hat{x}_{k}^{N G}-\beta_{k}^{N G}\right)+\left(\beta_{k}^{N G}-\tilde{\beta}_{k}^{G}\right)
$$

which implies

$$
\left|\hat{x}_{k}^{N G}-\tilde{\beta}_{k}^{G}\right|^{2} \leq\left[\left|\hat{x}_{k}^{N G}-\beta_{k}^{N G}\right|+\left|\beta_{k}^{N G}-\tilde{\beta}_{k}^{G}\right|\right]^{2}
$$

Now, one can apply Minkowski's inequality to obtain

$E\left|\hat{x}_{k}^{N G}-\tilde{\beta}_{k}^{G}\right|^{2} \leq\left[\sqrt{E\left|\hat{x}_{k}^{N G}-\beta_{k}^{N G}\right|^{2}}+\sqrt{E\left|\beta_{k}^{N G}-\tilde{\beta}_{k}^{G}\right|^{2}}\right]^{2}$

Also, from Remark 4.6 and (34), we have

$$
E\left|\hat{x}_{k}^{N G}-\beta_{k}^{N G}\right|^{2} \leq M_{\sigma_{1}} M_{z} \exp \left(-2 \sigma_{\gamma} k\right)
$$

where $0<\sigma_{\gamma}=\sigma_{1}-\sigma_{d}$.

Now, consider the process $\tilde{e}_{k} \triangleq \beta_{k}^{N G}-\tilde{\beta}_{k}^{G}$. Using (25), (16), one can write

$$
\begin{aligned}
\tilde{e}_{k}= & \left(F-\widetilde{\Sigma}_{k} H^{\prime} H F\right) \tilde{e}_{k-1} \\
& -\mu \widetilde{\Sigma_{k}} \Sigma_{w}^{-1} F V_{k-1} \Phi_{k-1} D_{k-1}\left(N_{k-1}^{N G}\right), \tilde{e}_{0}=0
\end{aligned}
$$

Denoting $\mu \widetilde{\Sigma}_{k} \Sigma_{w}^{-1} F V_{k-1}$ as $R_{k-1}$, one can then obtain

$$
E\left|\tilde{e}_{k}\right|^{2}=E\left|\sum_{j=0}^{k-1} \widetilde{\Psi}_{k} \widetilde{\Psi}_{j+1}^{-1} R_{j} \Phi_{j} D_{j}\right|^{2}
$$

where $\widetilde{\Psi}_{k}$ is the transition matrix associated with $F-\widetilde{\Sigma}_{k} H^{\prime} H F$. Using the assumption that $\widetilde{\Sigma}_{k}$ is stable for all $k>0$, one can have $\sup _{k}\left\|R_{k}\right\|<\infty$ which implies

$$
\begin{aligned}
E\left|\tilde{e}_{k}\right|^{2} & \leq K_{a} E\left[\sum_{j=0}^{k-1}\left\|\widetilde{\Psi}_{k} \widetilde{\Psi}_{j+1}^{1}\right\|\left|\Phi_{j} \|\right| D_{j} \mid\right]^{2} \\
& \leq M_{\sigma, \sigma_{1}} E\left[\sum_{j=0}^{k-1} \exp [-\sigma(k-j-1)] \exp \left(-\sigma_{1} j\right)\left|D_{j}\right|\right]^{2} \\
& \leq M_{\sigma, \sigma_{1}}^{a} k E\left[\sum_{j=0}^{k-1} \exp [-2 \sigma(k-j)] \exp \left(-2 \sigma_{1} j\right)\left|D_{j}\right|^{2}\right] \\
& \leq M_{\sigma, \sigma_{1}}^{a} M_{z} k \exp (-2 \sigma k)\left[\sum_{j=0}^{k-1} \exp \left[2\left(\sigma-\sigma_{\gamma}\right) j\right]\right] \\
& \leq M_{\sigma, \sigma_{1}, \sigma_{\gamma}} M_{z} k \exp \left(-2 \sigma_{\gamma} k\right)\left[1-\exp \left[-2\left(\sigma_{-} \sigma_{\gamma}\right) k\right]\right] \\
& \leq M_{\sigma, \sigma_{1}, \sigma_{\gamma}} M_{z} k \exp \left(-2 \sigma_{\gamma} k\right)
\end{aligned}
$$

where we have assumed $\sigma>\sigma_{\gamma}$ If $\sigma<\sigma_{\gamma}$ we have the following expression for the above bound

$$
E\left|\tilde{e}_{k}\right|^{2} \leq M_{\sigma, \sigma_{1}, \sigma_{\gamma}}^{b} M_{z} k \exp (-2 \sigma k)
$$

In the above derivation, the exponential stability of $\widetilde{\Psi}_{k} \widetilde{\Psi}_{j+1}^{-1}$ has been used following (14). Also, $K_{a}, M_{\sigma, \sigma_{1}}, M_{\sigma, \sigma_{1}}^{a}$, $M_{\sigma, \sigma_{1}, \sigma_{\gamma^{\prime}}} M_{\sigma, \sigma_{1}, \sigma_{\gamma}}^{b}$ are constants independent of $k$. We have also used the so-called $C_{r}$-inequality (Chen and Guo [10]) which states

$$
E\left(\sum_{j=1}^{n}\left|u_{j}\right|\right)^{r} \leq n^{r-1} E \sum_{j=1}^{n}\left|u_{j}\right|^{r}, r \geq 1
$$

It is clear from the above that as $k \rightarrow \infty$, we have $E\left|\tilde{e}_{k}\right|^{2} \rightarrow$ 0 and $E\left|\hat{x}_{k}^{N G}-\beta_{k}^{N G}\right|^{2} \rightarrow 0$. Combining these two results, we have (35).

Remark 4.7 One can prove a corollary similar to Corollary 3.1 stating $E\left|\hat{x}_{k}^{N G}-\beta_{k}^{N G}\right|^{2} \rightarrow 0$ as $k \rightarrow \infty$.

\section{CONCLUSIONS}

In this paper, we investigated the problem of asymptotic forgetting of initial conditions by risk-sensitive filters for linear time-invariant systems. For Gaussian initial conditions, we show that under an asymptotic stability condition satisfied by a state transition matrix associated with the $H_{\infty}$-like Riccati difference equation, with appropriate stabilizability and detectability condition holding for the linear system under consideration, the optimal risksensitive estimate initialized with the true initial covariance matrix approaches a suboptimal risk-sensitive estimate initialized with an incorrect covariance matrix in the mean square sense. For non-Gaussian initial conditions, the analysis is more complex. However, under a certain boundedness condition satisfied by the fourth order abso- 
lute moment of the initial state distribution and a slow growth condition satisfied by a certain Radon-Nikodym derivative, we have a similar mean square convergence result.

\section{REFERENCES}

1. Atar, R. and O. Zeitouni, "Exponential Stability for Nonlinear Filtering," Preprint (1996).

2. Atar, R. and O. Zeitouni, "Lyapunov Exponents for Finite-State Nonlinear Filtering," SIAM J. Contr. Optimiz, Vol. 35, No. 1, pp. 36-55 (1997).

3. Benes, V., "Exact Finite-Dimensional Filters for Certain Diffusions with Nonlinear Drift," Stochastics, Vol. 5, pp. 65-92 (1981).

4. Bensoussan, A., Stochastic Control of Partially Observable Systems, Cambridge University Press, Cambridge, UK (1992).

5. Bitmead, R.R., M. Gevers and V. Wertz, Adaptive Optimal Control: The Thinking Man's GPC, PrenticeHall, Brunswick, Victoria, Australia (1990).

6. Boel, R.K., M.R. James and I.R. Petersen, "Robustness and Risk Sensitive Filtering," in Proc. Conf. Decis. Contr., San Diego PP. 2273-2278 (1997).

7. Budhiraja, A. and D. Ocone, "Exponential Stability of Discrete-Time Filters for Bounded Observation Noise," Syst. Contr. Lett., Vol. 30, pp. 185-194.

8. Charalambous, C.D., "The Role of Information State and Adjoint in Relating Nonlinear Output Feedback Risk-Sensitive Control and Dynamic Games," IEEE Trans. Automat. Contr., Vol. 42, No. 8, pp. 1163-1170 (1997).

9. Charalambous, C.D., S. Dey and R.J. Elliott, "New Finite-Dimensional Risk-Sensitive Filters: Smallnoise Limits," IEEE Trans. Automat. Contr., Vol. 43, pp. 1424-1429 (1998).

10. Chen, H.F. and L. Guo, Identification and Stochastic Adaptive Control, Birkhauser, Boston (1991).

11. Dey, S. and C.D. Charalambous, "On Asymptotic Stability of Continuous-Time Risk-Sensitive Filters with Respect to Initial Conditions," Submitted (1997).

12. Dey, S., R.J. Elliott and J.B. Moore, "Finite-Dimensional Risk-Sensitive Estimation for Continuous-Time Nonlinear Systems," in Proc. Eur. Contr. Conf., Brussels (1997).

13. Dey, S. and J. Moore, "Risk-Sensitive Filtering and Smoothing for Hidden Markov Models," Syst. Contr. Lett., Vol. 25, No. 5, pp. 361-366.

14. Dey, S. and J. Moore, "Risk-Sensitive Filtering and Smoothing Via Reference Probability Methods," IEEE Trans. Automat. Contr., Vol. 42, No. 11, pp. 1587 1591 (1997).

15. Deylon, B. and O. Zeitouni, "Lyapunov Exponents for Filtering Problems. in Applied Stochastic Analysis Davis, M. and Elliott, R., Eds., pp. 511-535, Gordon and Breach, New York (1991).
16. Elliott, R.J., L. Aggoun and J.B. Moore, Hidden Markov Models: Estimation and Control, SpringerVerlag, New York (1994).

17. James, M., J. Baras and R. Elliott, "Risk-Sensitive Control and Dynamic Games for Partially Observed Discrete-Time Nonlinear Systems," IEEE Trans. Automat. Contr., Vol. 39, No. 4, pp. $780-792$ (1994).

18. Makowski, A.M., "Filtering Formulae for Partially Observed Linear Systems with Non-Gaussian Initial Conditions," Stochastics, Vol. 16, pp. 1-24 (1986).

19. Moore, J.B., R.J. Elliott and S. Dey (1995), "RiskSensitive Generalizations of Minimum Variance Estimation and Control," in IFAC Symp. Nonlin. Contr. Syst. Design, pp. 465-470, Tahoe City, CA. Also see summary in J. Math. Syst. Estimat. Contr., Vol. 7, No. 1, pp. 123-126 (1997).

20. Ocone, D. and E. Pardoux, "Asymptotic Stability of the Optimal Filter with Respect to Its Initial Conditions," SIAM J. Contr. Optimiz., Vol. 34, No. 1, pp. 226-243 (1996).

21. Ocone, D.L, "Asymptotic Stability of Benes Filters," Preprint (1998).

22. Sowers R.B. and A.M., "Discrete-Time Filtering for Linear Systems with Non-Gaussian Initial Conditions: Asymptotic Behaviour of the Difference between the MMSE and LMSE Estimates," IEEE Trans. Automat. Contr., Vol. 37, No. 1, pp. 114-120 (1992).

23. Speyer, J., C. Fan and R. Banavar, "Optimal Stochastic Estimation with Exponential Cost Criteria," in Proc. 31st Conf. Decis. Contr., Tucson, AZ, Vol. 2, pp. 2293-2298 (1992).

24. Whittle, P., "Risk-Sensitive Linear/Quadratic/ Gaussian Control," Adv. Appl. Probab., Vol. 13, pp. 764-777 (1981).

25. Whittle, P., “A Risk-Sensitive Maximum Principle: The Case of Imperfect State Observations," IEEE Trans. Automat. Contr., Vol. 36, No. 7, pp. 793-801 (1991).

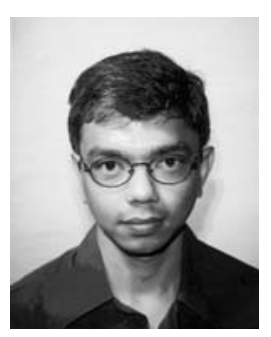

Subhrakanti Dey was born in Calcutta, India in 1968. He obtained his Bachelor of Technology and Master of Technology Degree from the Dept. of Electronics and Electrical Communication Engineering, Indian Institute of Technology, Kharagpur, India, in 1991 and 1993, respectively. He obtained his Doctor of Philosophy degree from the Dept. of Systems Engineering, Research School of Informations Sciences and Engineering in the Australian National University, Canberra, Australia in 1996. He is currently appointed as a Lecturer with the Department of Electrical and Electronic Engineering in the University of Melbourne since February 2000. During September 1995- 
September 1997 and September 1998-February 2000, he was appointed as a postdoctoral Research Fellow in the Department of Systems Engineering, Australian National University. During September 1997-September 1998, he was a post-doctoral Research Associate at the Institute for Systems Research, University of Maryland, College Park, U.S.A. His current research interests include signal processing for telecommunications, wireless communications and networks, performance analysis of communication networks, stochastic and adaptive estimation and control and statistical and adaptive signal processing. He is currently an academic staff member of the ARC Special Research Centre for Ultra-Broadband Informamation Networks and Co-operative Research Centre for Sensor, Signal and Information Processing in the Department of Electrical and Electronic Engineering, University of Melbourne.

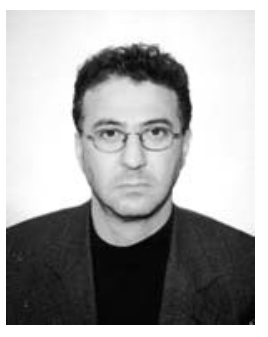

Charalambos D. Charalambous was born in Cyprus, on July 4, 1962. He received a Ph.D. degree from Old Dominion University, Norfolk, VA, in December 1992. Currently, he is an Associate Professor with University of Ottawa, School of Information Technology and Engineering, CA, and Adjunct Professor with McGill University, ECE Department, and an associate member of the Centre for Intelligent Machines. From 1995 to 1999, he was a visiting assistant professor with McGill University, ECE Department; from 1993 to 1995, he was a Post-Doctoral Research Fellow with Idaho State University, Engineering Department, Idaho. His research interests incluede stochastic processes and their applications in estimation, decision and control, robust control, information theory, and wireless communication at the physical and network level, and mathematical finance. 